\title{
RIKEN sees the light?
}

\section{Tokyo}

WiTH the opening last month of a new centre for photodynamics research near Sendai in northern Japan, the Institute of Physical and Chemical Research (RIKEN) has again proved itself to be an odd fish among Japan's many government-funded research laboratories. RIKEN's choice for director of the new institute is Junichi Nishizawa, one of the most outspoken critics of the Japanese research system in general and of the Ministry of Education, Science and Culture in particular.

The new institute is one of several initiatives with which RIKEN has tried to break away from the decaying facilities and ageing populations of researchers that plague government institutes and the national universities. Apart from the new centre in Sendai, RIKEN established a centre for life science research in Tsukuba, north of Tokyo, in 1984, and is now in the process of building the worla's most powerful synchrotron, SPring-8 (Super Photon Ring-8 GeV), at Harima Science Park City west of Osaka, in collaboration with the Japan Atomic Energy Research Institute.

In 1986 RIKEN introduced a novel programme, the Frontier Research Program, which employs teams of young researchers under short-term contracts to study the brain, new materials, and homeostasis (Nature 352, 569; 1987). Several of the teams are led by foreign scientists, something that is normally unheard of in Japan.

The new Photodynamic Research Centre in Sendai is part of the Frontier Research Program and will contain four laboratories: the laboratory for submillimetre waves, which will aim at developing new technology, in particular communication technology, for use in the range $100 \mathrm{GHz}$ to $1 \mathrm{THz}$; the laboratory for photophysics, which will focus on nonlinear interactions between light and inorganic materials, such as ionic crystals, with the ultimate aim of developing optical-computing devices; the laboratory of organometallic photodynamics for design of novel high-performance photofunctional materials; and the laboratory for photobiology, which will examine the physiological functions of light in living organisms.

The centre is expected to have an annual budget of close to $¥ 60(0)$ million (\$4.5 million) from the Science and Technology Agency (STA) from next fiscal year. The local prefectural and municipal governments in the Sendai area are providing land and a new building for the centre free of charge. Construction of the building is expected to begin shortly. Industry will also contribute "some signi100

ficant support" for research at the centre, according to Minoru Oda, director of RIKEN.

Oda attributes RIKEN's unusual adaptability to the institute's past history. It was established as a private foundation and, before the Second World War, received most of its income from patents on its own inventions. After the war RIKEN had to be radically re-organized because of anti-cartel laws introduced by JAPANESE UNIVERSITIES

\section{New money and new ideas}

\section{Tokyo}

JuNICII Nishizawa last week found himself both director of RIKEN's Photodynamics Research Centre and president of Tohoku University in Sendai a remarkable pair of posts for a man who has spent his research career collecting foreign prizes for his optoelectronics research and criticizing the uncreative atmosphere of Japanese universities and research institutes, an atmosphere which drove him to set up his own independent institute.

Among Nishizawa's prizes is the prestigious Jack A. Morton prize from the US Institute of Electrical and Electronic Engineers, which he received for the invention of the static induction transistor.

In a recent interview in the Japanese monthly magazine Voice he lambasted Japanese university researchers for "heading in the wrong direction" by rushing to work on discoveries made in the West "just as a new gold discovery attracts thousands of prospectors who all start to dig in the same place". In Nishizawa's view, success in basic research can be achieved only by "patiently digging an area no one has ever dug before".

Now that he has joined the establishment, he still intends to find his way around the conservative Ministry of Education, Science and Culture (MESC) by using the new RIKEN institute to help bring non-MESC money to the university. Nishizawa says that MESC was initially opposed to his idea of bringing the RIKEN photodynamics centre to Sendai. But he points out that he is simply returning RIKEN to its roots (RIKEN was provisionally established at Tohoku Imperial University before moving to its present site near Tokyo).

And he says that he has repeatedly told MESC that they should never have allowed the Science and Technology Agency (STA) to take over RIKEN after the war and that it was only because of "idleness" on their part that STA succeeded in gaining control of the institute. the US occupation forces (the US army also dumped RIKEN's cyclotrons in the sea on the grounds that they might be used to make nuclear weapons). Now RIKEN gets about 90 per cent of its funds from STA. Nevertheless, RIKEN still has comparative freedom to establish new laboratories and research programmes when compared with other government-funded research laboratories. Oda says STA gives RIKEN this freedom because "they have learned this is the best way".

David Swinbanks

Three out of five professors at the new centre will be from Tohoku University, and the other two will come from the Wako campus of RIKEN. The centre will have a total complement of about 30 researchers, Nishizawa says.

In addition to the new centre. Nishizawa hopes to bring other STA money to the university, for example through STA's ERATO programme (Nishizawa himself has held two ERATO awards). And he is also making arrangements for industry to fund new chairs at the university. Tokyo University already has several such positions (Nature 348, 7; 1990)

But unlike the chairs at Tokyo, which are primarily short-term contract positions for foreigners, Nishizawa intends to create long-term posts for Japanese professors.

Nishizawa says he is also strongly proposing to MESC that the ministry should double present funds for competitive research grants to about $¥ 100,000$ million a year (a proposal that has also been put forward by other Japanese academics -see Nature 339, 575; 1989). He says that many people agree with him, including powerful people in the Ministry of Finance and the Diet, but MESC has yet to accept his proposal.

With the new money Nishizawa says he hopes to promote research that relies on "modest serendipity", like that which led to the discovery of high-temperature super-conductors at IBM Zurich, rather than ploughing money into buying expensive new equipment as Japanese professors are apt to do. He says the United Kingdom is a model of such research and he would like Japan to learn from the UK system.

But to promote creative research of the type envisioned by Nishizawa requires not only money but a complete overhaul of the organization of the university research system, in order to release young researchers from the control of professors.

David Swinbanks 